AUDIT

\title{
Iron deficiency anaemia: are the British Society of Gastroenterology guidelines being adhered to?
}

\section{R N Patterson, S D Johnston}

See end of article for authors' affiliations

\section{Correspondence and} reprint requests to: Dr Neil Patterson, Department of Medicine, Mulhouse

Building, Royal Victoria

Hospital, Grosvenor Road, Belfast BT1 2 6BJ, Northern Ireland;

neil@rnpatterson.idps.co.uk

Submitted

10 January 2002

Accepted

18 February 2003
Background: The British Society of Gastroenterology (BSG) issued guidelines on the investigation of iron deficiency anaemia (IDA) ensuring standardised and comprehensive gastrointestinal investigation in all patients. It was apparent that not all patients in the authors' hospital were investigated according to these guidelines.

Objective: To determine whether patients who were referred for upper gastrointestinal endoscopy for investigation of IDA were confirmed to be iron deficient, and whether the BSG guidelines were being fully implemented.

Methods: All patients referred for upper gastrointestinal endoscopy over an 18 month period on a computer database (Endoscribe) were reviewed. Haematology, biochemistry, and radiology results were obtained and the frequency of the various diagnoses recorded.

Results: A total of 320 patients (133 male; mean age 71.5 years) were initially referred for upper gastrointestinal endoscopy for investigation of IDA, of whom 95 were iron deficient. Of these, 44 $(46 \%)$ had duodenal biopsies performed, three $(7 \%)$ of whom were diagnosed with coeliac disease. Five patients were diagnosed with upper gastrointestinal carcinoma (one oesophageal, four gastric). Of the remaining 87 patients, $65(75 \%)$ underwent lower gastrointestinal investigations with four having colorectal carcinoma, four colonic polyps, and one angiodysplasia.

Conclusions: Duodenal biopsies were performed in less than half of the patients. In those not diagnosed with coeliac disease or upper gastrointestinal carcinoma, only three quarters underwent lower gastrointestinal assessment. Approximately 10\% were diagnosed with gastrointestinal malignancy as a cause for their anaemia and in $66 \%$ of patients no gastrointestinal cause was found. All physicians need to be made fully aware of the BSG guidelines for investigation of IDA. p on deficiency anaemia (IDA) is a common problem both in primary and secondary care. Estimates of the prevalence of IDA in adults vary widely depending on the population screened, although it is believed to affect between $2 \%-5 \%$ of adults. $^{12}$ Gastrointestinal blood loss has been found to account for anaemia in $27 \%-95 \%$ of cases and is the commonest cause of IDA in men and postmenopausal women. ${ }^{13-6}$ Despite adequate investigation, no clear cause for anaemia is identified in $10 \%-43 \%$ of patients. ${ }^{78}$ One previous study has highlighted that there is substantial under investigation of IDA, with serious treatable conditions being undetected.' Subsequently, the British Society of Gastroenterology (BSG) produced guidelines for the investigation of IDA in $2000 .{ }^{10}$ It was proposed that all adult male patients and postmenopausal female patients, without overt blood loss or any other obvious cause of IDA, should undergo upper gastrointestinal endoscopy, including duodenal biopsy, and colonoscopy or barium enema, with or without flexible sigmoidoscopy.

The aim of this study was to determine if patients initially referred for upper gastrointestinal endoscopy for evaluation of anaemia did in fact have documented iron deficiency, and to determine whether appropriate upper and lower gastrointestinal investigations were performed, in accordance with the BSG guidelines for the investigation of IDA.

\section{METHODS}

All patients undergoing upper gastrointestinal endoscopy for the investigation of anaemia, between September 2000 and February 2002, were identified from a computerised endoscopy database (Endoscribe). Haematology results were reviewed to determine if IDA was present. Anaemia was defined as haemoglobin concentration $<11.5 \mathrm{~g} / \mathrm{dl}$ in women and haemoglobin concentration $<12.5 \mathrm{~g} / \mathrm{dl}$ in men (representing the lower limits of our hospital reference laboratory). Definite iron deficiency was defined as serum ferritin $<12$ $\mu \mathrm{g} / \mathrm{dl}$. Probable iron deficiency was defined as mean corpuscular volume $<76 \mathrm{fl}$ (lower range of hospital reference laboratory), in association with appropriate changes in serum iron, total iron binding capacity, and percentage iron saturation, when the serum ferritin was $>12 \mu \mathrm{g} / \mathrm{dl}$. Patients were excluded if they did not fulfil the definition for definite IDA or probable iron deficiency. Patients with a history of previous oesophageal varices or symptoms of haematemesis or melaena to suggest overt gastrointestinal blood loss were also excluded.

The medical records of patients with definite and probable iron deficiency were assessed to determine if they had a duodenal biopsy performed at the time of upper gastrointestinal endoscopy. In accordance with the BSG guidelines, if patients did not have upper gastrointestinal malignancy or coeliac disease diagnosed at the time of upper gastrointestinal endoscopy, patients' records were assessed to determine whether a colonoscopy, flexible sigmoidoscopy or barium enema was performed. The following were considered as aetiological diagnoses: coeliac disease, upper and lower gastrointestinal carcinoma, erosive gastritis, erosive oesophagitis (grade III and IV), peptic ulcer, colonic polyps, and angiodysplasia. The frequency of the various diagnoses were recorded.

Abbreviations: BSG, British Society of Gastroenterology; IDA, iron deficiency anaemia 
Table 1 Results of lower gastrointestinal

investigations: a comparison of barium enema and colonoscopy

\begin{tabular}{|c|c|c|c|}
\hline Diagnosis & $\begin{array}{l}\text { Barium enema } \\
(n=50)\end{array}$ & $\begin{array}{l}\text { Colonoscopy } \\
(n=15)\end{array}$ & $\mathrm{p}$ Value \\
\hline Diverticular disease & 24 & 4 & 0.24 \\
\hline Colonic polyps & 1 & 3 & 0.05 \\
\hline Colonic carcinoma & 3 & 1 & 0.6 \\
\hline Normal investigations & 12 & 5 & 0.89 \\
\hline Angiodysplasia & 0 & 1 & NA \\
\hline Did not attend & 7 & 0 & NA \\
\hline Inflammatory stricture & 1 & 0 & NA \\
\hline Technically difficult & 1 & 1 & 0.4 \\
\hline Results unobtainable & 1 & 0 & NA \\
\hline Total & 50 & 15 & \\
\hline
\end{tabular}

\section{RESULTS}

Three hundred and twenty patients ( 133 male; mean age 71.5 years) underwent upper gastrointestinal endoscopy during the study period for evaluation of iron deficiency anaemia. Of these, 40 patients ( 22 male; mean age 65 years) had symptoms of haematemesis or melaena, 112 (49 male; mean age 74.8 years) had no evidence of iron deficiency, 63 (23 male; mean 70.3 years) had no serum ferritin recorded, and 10 (one male; mean age 49.7 years) did not fit the definition for anaemia, leaving 95 patients ( 37 male; mean age 68.5 years) in the study group. This group included 19 patients (nine male; mean 69.5 years) who met the definition for probable iron deficiency. The mean haemoglobin concentration was $8.6 \mathrm{~g} / \mathrm{dl}$ (range 5.1-12.4) in the male patients and $8.7 \mathrm{~g} / \mathrm{dl}$ in the female patients (range 4.3-11.4).

Forty four patients ( 16 male; mean age 66.2 years) had a duodenal biopsy performed at the time of upper gastrointestinal endoscopy, representing $46 \%$ of the study group. In three cases $(7 \%)$ duodenal biopsy demonstrated either total or subtotal villous atrophy in keeping with coeliac disease. Five $(5 \%)$ patients were diagnosed with upper gastrointestinal carcinoma, one of whom (male aged 59 years) had oesophageal carcinoma and four (all male; mean age 62 years) had gastric carcinoma. In addition, four patients had erosive oesophagitis (at least grade III), one had erosive gastritis, seven had a gastric ulcer (one of whom had coeliac disease), and two had a duodenal ulcer. In total, 21 of 95 patients $(22 \%)$ undergoing upper gastrointestinal endoscopy had an aetiology identified to explain their IDA.

Of the 87 patients ( 32 male; mean age 69.1 years) who were not diagnosed with coeliac disease or upper gastrointestinal cancer at upper gastrointestinal endoscopy, 65 underwent lower gastrointestinal investigations, representing $75 \%$ of the study group. Of these, 15 (six male; mean age 67.6 years) (17\%) had colonoscopy, 50 (18 male; mean age 70 years) (57\%) had a barium enema arranged, and one patient had both flexible sigmoidoscopy and barium enema. The main aetiologies identified after lower gastrointestinal investigations were colonic polyps $(n=4)$, colorectal carcinoma $(n=4)$, and angiodysplasia $(n=1)$ (table 1$)$. Of those patients $(n=15)$ who underwent colonoscopy, six $(40 \%)$ had a potential attributable cause for the anaemia found compared with only three $(6 \%)$ of those who underwent barium enema $(n=50)$ $(\mathrm{p}<0.01)$. Regarding the individual diagnoses, the detection rates did not differ significantly between colonoscopy and barium enema (table 1). Table 2 lists the final diagnoses made after completed gastrointestinal investigations for IDA.

\section{DISCUSSION}

Of the 320 patients initially identified, only 95 met our inclusion criteria for definite or probable iron deficiency. We
Table 2 Final diagnoses made after upper with or without lower gastrointestinal investigations

\begin{tabular}{ll}
\hline Diagnosis & No of patients \\
\hline Oesophagitis (at least grade III) & 4 \\
Oesophageal carcinoma & 1 \\
Gastritis (erosive) & 1 \\
Gastric ulcer & $7^{*}$ \\
Gastric carcinoma & 4 \\
Duodenal ulcer & 2 \\
Coeliac disease & $3 *$ \\
Colonic polyps & 4 \\
Colonic carcinoma & 4 \\
Angiodysplasia & 1 \\
No cause found & 63 \\
Others & $2 \dagger$ \\
\hline \multirow{2}{*}{ *One patient had coeliac disease and a gastric } \\
ulcer. \\
†Others: "Creon" induced inflammatory stricture; \\
stricture at splenic flexure diagnosed on computed \\
tomography. No lower gastrointestinal \\
investigation.
\end{tabular}

excluded 185 patients who were referred for investigation of anaemia who were not confirmed to be iron deficient. Given that the BSG guidelines are specifically for the investigation of patients with IDA, the importance of measuring haematinics to correctly characterise the type of anaemia on all patients before undertaking further investigations is a fundamental first step. This information should be included in the referral for upper gastrointestinal endoscopy. As a result unnecessary investigations may be avoided. In addition, patients were only included in this audit if the referring physician had decided that it was appropriate to investigate IDA in their patient. Clearly there will be patients with IDA in whom it is not deemed appropriate to embark on investigations of IDA-for example, due to confounding factors such as age or extensive comorbidity. From our laboratory system it is not possible to obtain an estimate of the number of patients with IDA who were not referred for gastrointestinal investigations.

In our study only $46 \%$ of patients undergoing upper gastrointestinal endoscopy for the investigation of IDA had duodenal biopsies performed. Since the prevalence of coeliac disease in Northern Ireland is 1:122 in the general population, ${ }^{11}$ this finding is disappointing. Of those patients who had duodenal biopsy performed, $7 \%$ had histological features of coeliac disease, reinforcing the importance of routine biopsies in all patients referred for investigation of IDA, in accordance with the BSG guidelines.

In total $21(22 \%)$ patients had a cause identified for their anaemia at upper gastrointestinal endoscopy, of whom eight had either neoplasia or coeliac disease. Only $75 \%$ of the remaining patients proceeded to lower gastrointestinal investigations. In nine patients (14\%) a cause was found for the anaemia. Colonoscopy as a diagnostic tool for investigation of iron deficiency yielded a higher proportion of positive diagnoses than barium enema. This predominantly relates to the superior detection of colonic polyps and angiodysplasia by colonoscopy.

If a cause for IDA is not found at the time of upper gastrointestinal endoscopy, we would advise the referring physician to proceed with lower gastrointestinal investigations. The reason why one quarter of our patients did not proceed to lower gastrointestinal investigations is not clear. Their age did not differ significantly from those who underwent lower gastrointestinal investigations; however, comorbidity or ignorance of the BSG guidelines for the adequate investigation of anaemia are possible explanations.

In $63(66 \%)$ of the 95 patients no cause was found for the IDA, after upper and lower gastrointestinal investigations. The 
BSG guidelines state that such patients should be monitored three monthly for one year with additional iron replacement being given if the haemoglobin or mean cell volume falls below normal, with consideration of further investigations only if the haemoglobin concentration cannot be maintained. Reassuringly, follow up studies in patients with negative gastrointestinal investigations confirm that iron deficiency does not return and has a benign course in most patients. ${ }^{12}{ }^{13}$

In summary, a large proportion of patients referred for investigation of anaemia were not confirmed to be iron deficient. Even in those patients in whom investigations were considered appropriate, the BSG guidelines are not being fully implemented with only three quarters of patients proceeding to lower gastrointestinal investigations. Colonoscopy as a diagnostic tool for investigation of iron deficiency yielded a higher proportion of diagnoses which could be contributing to the IDA than barium enema. Approximately 10\% of our patients had a diagnosis of gastrointestinal malignancy as a cause of their IDA.

In conclusion, all physicians need to fully aware of the BSG guidelines for investigation of IDA, both in terms of appropriate referral and the necessary investigation pathway. Clearly this would need to be the subject of a further audit after a period of re-education and implementation of the BSG guidelines.

\section{Authors' affiliations}

R N Patterson, S D Johnston, Department of Gastroenterology, Belfast City Hospital, Northern Ireland, UK

\section{REFERENCES}

1 Calvey HD, Castledon CM. Gastrointestinal investigations for anaemia in the elderly: a prospective study. Age Ageing 1987;16:399-404.

2 Sayer JM, Long RM. Prospective survey of investigations in outpatients referred with iron deficiency anaemia. Gut 1993:34:1297-9.

3 Cook IJ, Pavli P, Riley JW, et al. Gastrointestinal investigation of iron deficiency anaemia. BM 1986;292:1380-2.

4 Ward MC, Gundroo D, Bailey RJ, et al. Effect of investigation of the management of elderly patients with iron deficiency anaemia. Age Ageing 1990; 19:204-6

5 Tan CC, Guan R, Tay HH, et al. The diagnostic yield of upper gastrointestinal endoscopy in the investigation of anaemia. Singapore Med J 1991;32:157-9.

6 Hershko C, Vitells A, Braverman DZ. Causes of iron-deficiency anaemia in an adult population. Blut 1984;49:347-52.

7 McIntyre As, Long RG. Prospective survey of investigations in outpatients referred with iron deficiency anaemia. Gut 1993;34:1 102-7.

8 Gordon S, Smith R, Power G. The role of endoscopy in the evaluation of iron deficiency anaemia in patients over the age of 50. Am J Gastroenterol 1994;89: 1963-7.

9 Lucas CA, Logan ECM, Logan RFA. Audit of the investigation and outcome of iron-deficiency anaemia in one health district. $J R$ Coll Physicians Lond 1996;30:33-6

10 Goddard AF, Mclntyre AS, Scott BB. Guidelines for the management of iron deficiency anaemia. British Society of Gastroenterology. Gut 2000;46:IV 1-5.

11 Johnston SD, Watson RG, McMillan SA, et al. Prevalence of coeliac disease in Northern Ireland. Lancet 1997;350:1370.

12 Sahay R, Scott BB. Iron deficiency anaemia-how far to investigate? Gut 1993;34:1427-8.

13 Gordon S, Benson S, Smith R. Long-term follow up of older patients with iron- deficiency anaemia after a negative $\mathrm{Gl}$ investigation. Am J Gastroenterol 1996;91:885-9

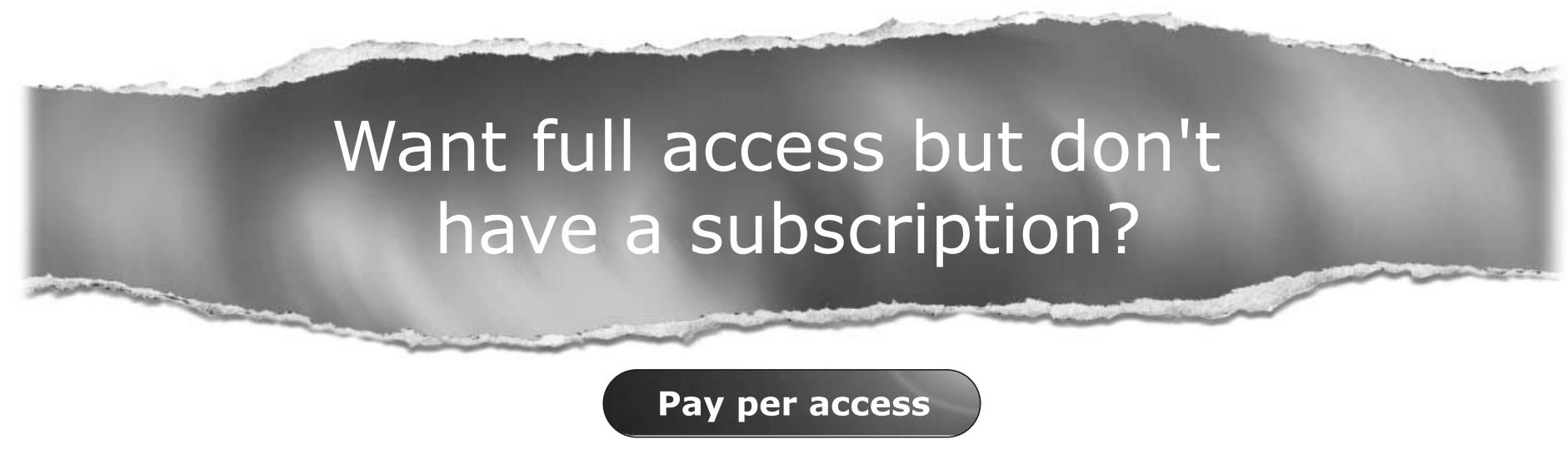

For just US\$25 you can have instant access to the whole website for 30 days. During this time you will be able to access the full text for all issues (including supplements) available. You will also be able to download and print any relevant pdf files for personal use, and take advantage of all the special features Postgraduate Medical Journal online has to offer. 